\title{
Controllable Cooperative Self-Assembly of PS-b-PAA/PS-b-P4VP Mixture by Tuning the Intercorona Interaction
}

\author{
Zhen Geng, ${ }^{\dagger, \ddagger}$ Zhongkai Cheng,§ Yutian Zhu,*,† and Wei Jiang*,†
}

†State Key Laboratory of Polymer Physics and Chemistry, Changchun Institute of Applied Chemistry, Chinese Academy of Sciences, Changchun 130022, People's Republic of China

‡University of Chinese Academy of Sciences, Beijing 100049, People’s Republic of China

§School of Life Sciences, Jilin University, Changchun 130022, People’s Republic of China 


\section{Supporting figures}
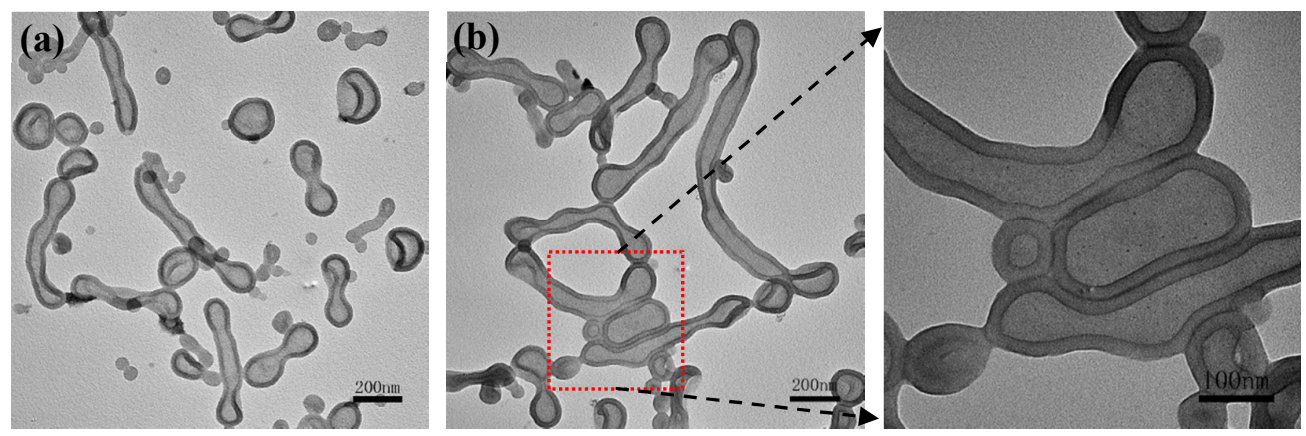

Figure S1. TEM images of vesicles from equimolar mixture of PS- $b$-P4VP and PS- $b$-PAA: (a) unstained samples, (b) stained by $I_{2}$ vapor before TEM observation. 

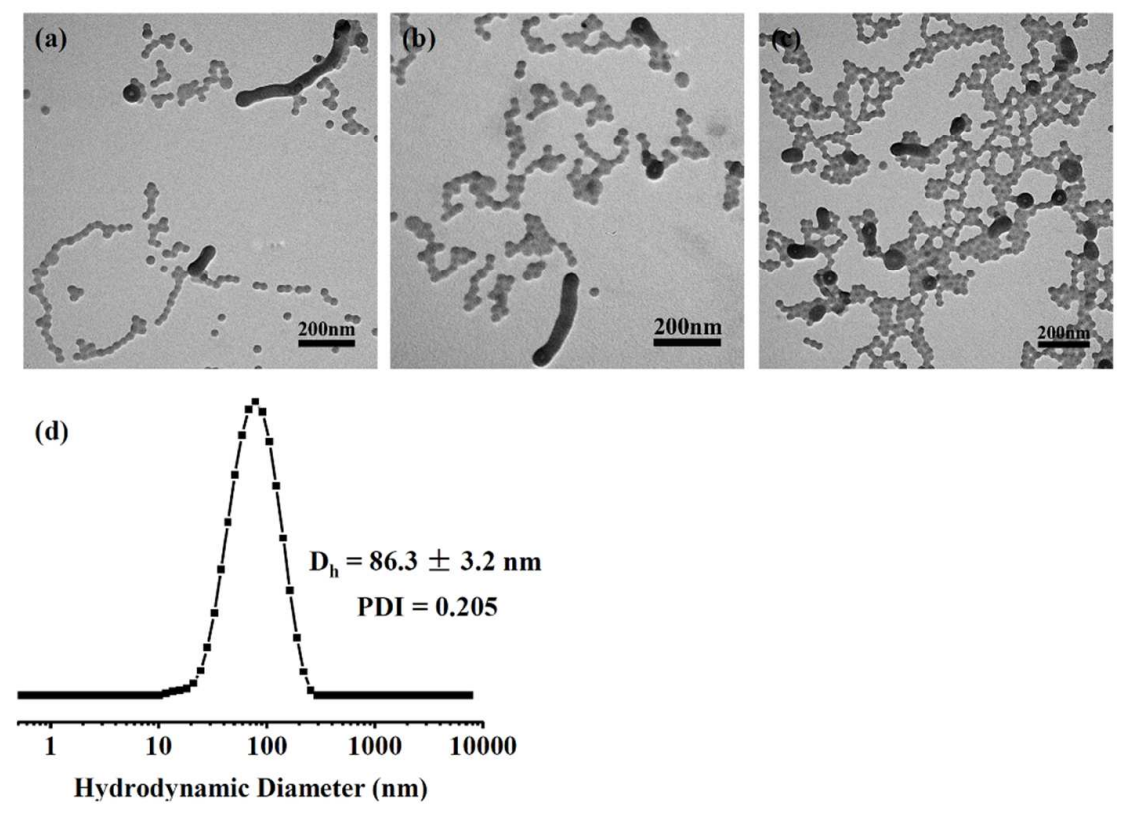

Figure S2. TEM images $(\mathrm{a} \sim \mathrm{c})$ and DLS result $(\mathrm{d})$ for the micelles of PS- $b$-P4VP/PS- $b$-PAA mixture at $\mathrm{R}=0.04, \mathrm{R}=\mathrm{NaOH} / \mathrm{AA}$. 

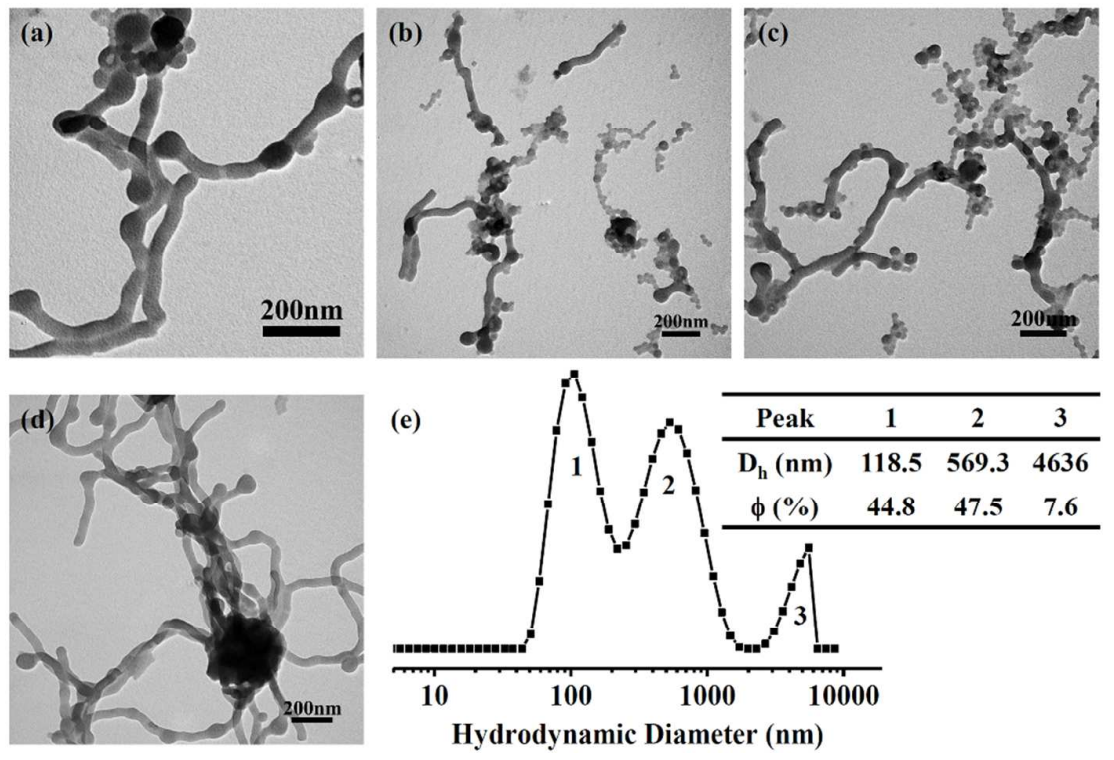

Figure S3. TEM images $(\mathrm{a} \sim \mathrm{d})$ and DLS result $(\mathrm{e})$ for the micelles of PS- $b$-P4VP/PS- $b$-PAA mixture at $\mathrm{R}=0.008, \mathrm{R}=\mathrm{NaOH} / \mathrm{AA}$. 

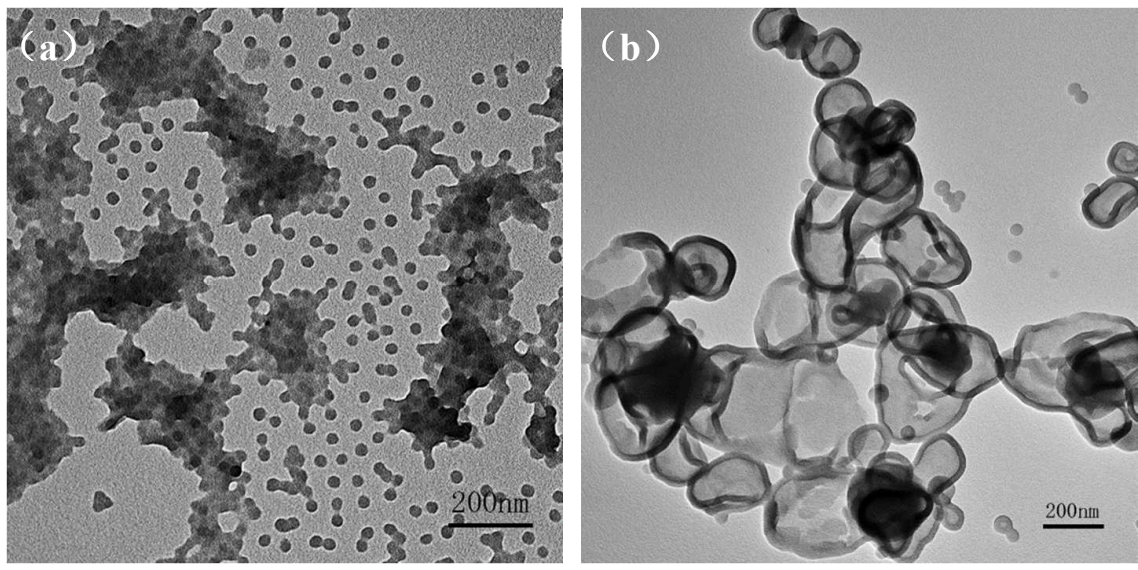

Figure S4. TEM images of aggregates from equimolar mixture of PS- $b$-P4VP and PS- $b$-PAA: (a) addition of $\mathrm{NaOH}$, mole ratio of $\mathrm{NaOH} / \mathrm{AA}, \mathrm{R}=4.8$, (b) addition of $\mathrm{HCl}$, mole ratio of $\mathrm{HCl} / 4 \mathrm{VP}, \mathrm{R}=0.00056$. 

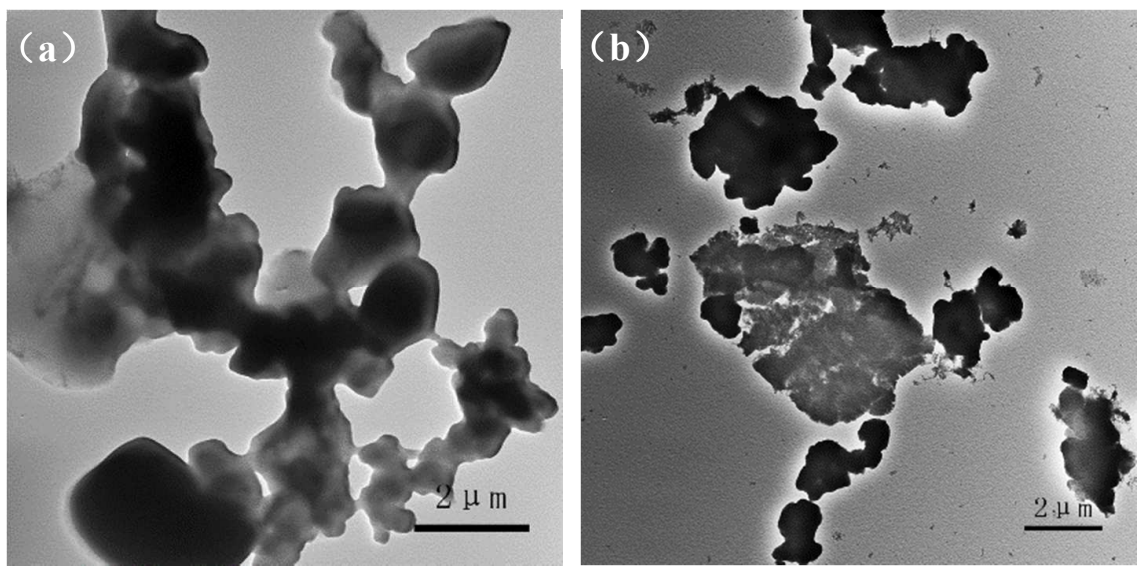

Figure S5. TEM images of irregular aggregates formed from (a) $\mathrm{PS}_{144}-b-\mathrm{PAA}_{22}$, the $\mathrm{NaOH} / \mathrm{AA}$ ratio $=4.8$ and (b) $\mathrm{PS}_{144}-b-\mathrm{P}_{4} \mathrm{VP}_{33}$, the $\mathrm{HCl} / 4 \mathrm{VP}$ ratio $=5.6$, in $\mathrm{DMF} /$ water $(66 / 34, v / v)$ 


\section{Complete author list for references with more than 10 authors:}

[1] Saiyin, W.; Wang, D. L.; Li, L. L.; Zhu, L. J.; Liu, B.; Sheng, L. J.; Li, Y. W.; Zhu, B. S.; Mao, L. M.; Li, G. L.; Zhu, X. Y. Sequential Release of Autophagy Inhibitor and Chemotherapeutic Drug with Polymeric Delivery System for Oral Squamous Cell Carcinoma Therapy. Mol. Pharm. 2014, 11, 1662-1675. (Reference 19 in manuscript.)

[2] Singh, M.; Odusanya, O.; Wilmes, G. M.; Eitouni, H. B.; Gomez, E. D.; Patel, A. J.; Chen, V. L.; Park, M. J.; Fragouli, P.; Iatrou, H.; Hadjichristidis, N.; Cookson, D.; Balsara, N. P. Effect of Molecular Weight on the Mechanical and Electrical Properties of Block Copolymer Electrolytes. Macromolecules 2007, 40, 4578-4585. (Reference 30 in manuscript.) 